\title{
"En defensa del sin par idioma de Cervantes": Historia de una tradición en la constitución de la disciplina escolar Lengua y Literatura
}

\author{
Valeria Sardi \\ (Universidad Nacional de La Plata. Argentina)
}

\begin{abstract}
Resumen
La conformación de la disciplina escolar Lengua y Literatura se constituye como una trama polifónica de múltiples dimensiones históricas, políticas, sociales y culturales. A su vez, su historia está ligada a la yuxtaposición y entrecruzamiento de tradiciones, paradigmas teóricos y modos de hacer que configuran el conocimiento escolar. En este artículo nos proponemos reconstruir desde una perspectiva histórica-cultural una tradición escolar que atraviesa la disciplina escolar Lengua y Literatura desde fines del siglo XIX hasta nuestros días: la defensa de una lengua escolar pura, sin contaminaciones. En esta tradición escolar ocupan un lugar central el uso de las gramáticas -como documentos donde se legisla la corrección idiomática- y la enseñanza de la normativa. Para ello indagaremos en diversidad de fuentes que permitirán reconstruir la experiencia cotidiana escolar: dispositivos didácticos como manuales, gramáticas; testimonios orales y propuestas de prácticas de lectura y escritura.
\end{abstract}

Palabras clave:

Disciplina Escolar - Lengua y Literatura - Tradición Escolar - Purismo Lingüístico Historia Cultural.

\section{Summary}

Language and Literature as a school discipline stands as a polyphony of multiple historical, political, social and cultural dimensions. Moreover, its history is related to the juxtaposition and intertwining of traditions, theoretical paradigms and ways of doing things that make up school knowledge. In this article we set out to re build from a historical and cultural perspective a school tradition that Language and Literature as a school discipline has gone through from the end of the XIX century to these days, namely, the defense of a pure, unpolluted school language. In this school tradition the use of grammars, documents which rule about idiomatic accuracy, and the teaching of normative grammar play a key role. To be able to re build the daily school experience 
we will look into several sources such as teaching aids like manuals and grammars, oral testimonies, and practical reading and writing proposals.

\section{Key words}

School Discipline - Language and Literature - School Tradition - Linguistic Purism Cultural History.

\section{Introducción}

En 1923 se publica en Buenos Aires un libro titulado Nuestra lengua del periodista y traductor platense Arturo Costa Álvarez en el que el autor plantea el presupuesto fundante de que el idioma nacional es el castellano "al que debemos limpiar de impurezas con que lo afean en la lengua vulgar la inmigración cosmopolita y en la lengua culta la lectura continua de libros extranjeros". Este alegato en defensa de una lengua pura -es decir, castiza, respetuosa de la normativa gramatical y sin elementos lingüísticos dialectales- se entrelaza con la admonición de los textos literarios ajenos al universo simbólico nacional que acechan en distintos rincones de la ciudad como los kioscos de revistas y otros espacios urbanos donde se traza el recorrido de la lectura de los sectores populares en ediciones económicas y traducciones no autorizadas -escena cultural que ya se escenificaba unos diez años antes a la publicación del libro de Costa Álvarez-. En un contexto urbano cambiante y de grandes transformaciones la cuestión inmigratoria cobra nuevas significaciones entre las que se encuentra la de corruptora de la idiosincrasia lingüística y cultural argentina; por lo tanto se impone como necesario borrar los habitus (1) de los recién llegados. La escuela, entonces, se erige como el espacio institucional donde conjurar esos temores sobre los que advierten, también, funcionarios ministeriales, inspectores, profesores y maestros. Y la disciplina escolar Lengua y Literatura se construye haciéndose eco de las prerrogativas sociales, políticas y culturales del contexto sociohistórico que determinan la constitución del currículum y de la enseñanza ya que imponen ciertos saberes y prácticas consideradas legítimas según el inconsciente cultural de la época. Esta relación entre sociedad y escuela da cuenta de cómo el currículum no puede pensarse como algo dado sino, más bien, se trata de una construcción sociocultural, fruto de una tradición selectiva que establece una selección de saberes y de prácticas. En este sentido, podemos decir con Ivor Goodson (2), que la disciplina es una invención social, cultural e histórica que se constituye en un microcosmos que da cuenta de la historia cultural de una sociedad; a su vez, se trata de un cuerpo de saber-poder, una serie de enunciados 
considerados verdaderos que establecen sus propias definiciones y reglas de juego y se insertan en cierto marco teórico. En ella no sólo se incluyen los saberes disciplinares sino también la formación de los sujetos, los dispositivos didácticos, las explicaciones de los docentes, la docimología, las planificaciones de clases, las prácticas de enseñanza, las experiencias de la cotidianeidad escolar. Por ello para su reconstrucción es necesario relevar diversidad de fuentes que den cuenta de las múltiples dimensiones que entran en juego; por un lado se considera el análisis de fuentes que remitan a las políticas educativas y a la burocracia escolar como digestos, circulares, programas, planes de estudio; por otro lado, publicaciones periódicas como revistas pedagógicas y escolares o periódicos que nos acerquen al contexto sociocultural investigado. También se analizan otras fuentes como los dispositivos pedagógicos; es decir, manuales, libros de lectura, antologías, gramáticas que nos permiten acceder a algunos de los textos leídos y a las consignas propuestas en el aula. Otro modo de acercamiento es a través de los propios protagonistas en entrevistas en profundidad a profesores o alumnos, o bien, analizando escrituras del yo como diarios y memorias de profesores que relatan la experiencia cotidiana escolar. Para esta historización, además, se pueden indagar fuentes icónicas que den cuenta de experiencias concretas o escenas de aula ya que permiten acrecentar la interpretación. Se trata de, a través de multiplicidad de fuentes, reconstruir la trama polifónica de la disciplina escolar.

Por otro lado, la disciplina escolar se constituye en el cruce, reemplazo y yuxtaposición de paradigmas, teorías, tradiciones escolares configuradas históricamente que ganan y pierden legitimidad en el campo educativo e intelectual de una comunidad de manera discontinua. Estas tradiciones de enseñanza y de conocimiento escolar son modos de hacer de los sujetos, esquemas de percepción interiorizados que se reproducen y se instituyen como prácticas en la memoria histórica de los docentes. Además, están ligadas a las dimensiones políticas e históricas y constituyen a la disciplina en un devenir que se caracteriza por los cambios, las transformaciones, los quiebres, las continuidades y discontinuidades.

\section{Historia de una tradición escolar}

La historia de la disciplina lengua y literatura en nuestro país está atravesada por tradiciones vinculadas a la enseñanza de la lengua y a la especificidad literaria que conforman el código disciplinar -entendido como una "tradición social configurada históricamente y compuesta de un conjunto de ideas, valores, suposiciones y rutinas que legitiman la función educativa atribuida (...) y que regulan el orden de la práctica de su enseñanza" (3)-. Una 
de estas tradiciones nace de la pregunta acerca de qué lengua enseñar en la escuela y la preocupación, ligada a este interrogante, por establecer una lengua escolar sin contaminaciones culturales y lingüísticas que pudieran diluir la identidad nacional. Esta tradición dialoga con los criterios de selección de los textos literarios -que también deben aportar a la construcción del "alma nacional"- que se pone en juego en el aula, con prácticas de lectura y escritura y con la utilización de determinados dispositivos didácticos. Un primer momento de esta tradición escolar podría datarse en el período entre siglos, en el momento en que se comienza a ampliar el sistema de aulas en nuestro país y se convoca a catedráticos españoles como autores de gramáticas y diccionarios, como funcionarios o bien como intelectuales al servicio del proyecto del nacionalismo cultural y como colaboradores para llevar a cabo la planificación lingüística monoglósica que se instaura para esa época. Una figura destacada en este contexto es Ricardo Monner Sans quien -desde su Gramática castellana (1893) de uso frecuente en las escuelas argentinas- apuesta a la necesidad de clasificar los "vicios de pronunciación" habituales en la Buenos Aires de la época que eran, según este autor, consecuencia inevitable del fenómeno inmigratorio y -como señala en De gramática y de lenguaje (1915)- a instaurar el uso de la gramática como instrumento imprescindible para fijar la lengua correcta ya que, considera, "la Gramática es esencialmente necesaria para aprender a hablar y escribir correctamente". Y, sentencia, "quien no sepa Gramática, tropezará siempre con los escollos que de continuo se alzan al pretender manifestar el pensamiento en forma correcta y bella" (4). Para su enseñanza propone el estudio de las reglas gramaticales que "nos advertirán sobre lo que no debemos decir para no caer en ridículo" y el estudio de los modelos literarios realizados por "maestros del buen hablar." Asimismo, Monner Sans advierte sobre las conspiraciones cotidianas contra el aprendizaje del idioma como libros que [...] se califican de modelos, trabajados en incorrectos trozos de deslavazada prosa; en las lecciones orales que en algunas clases recoge; en las conversaciones que doquier oye el joven mejor dispuestos para el estudio que nos ocupa, se desencanta o tiende a rebelarse contra lo legislado en materias de lenguaje (5) y que generan una situación anárquica en la que "hay que trocarse en héroe para no dejarse arrastrar por el aquilón demoledor". El idioma, según Monner Sans, corre peligro en un contexto cotidiano donde la lengua es cambiante, enriquecida por voces extranjeras y usos lingüísticos que no siguen las normas gramaticales como se puede ver en periódicos, revistas y libros baratos. En la escuela, entonces, es necesario pulir el lenguaje a través de ejercicios de pronunciación y ortografía -considerada como una ciencia que no puede modificarse de acuerdo a los 
usos vulgares- $y$, sobre todo, leer, estudiar y analizar "las bellezas esparcidas en libros clásicos, antiguos y modernos, procurando gustarlas y asimilárselas, a fin de no afear nuestra conversación ni nuestros escritos con giros contrarios a su genio y pureza". Además, considera que es fundamental tener en cuenta la etimología para aprender ortografía porque aquella nos permite conocer la historia y las transformaciones de la estructura de las palabras. La etimología, según Monner Sans, fija la norma ortográfica y "evita las corrupciones y mutilaciones" (6). Para este profesor, entonces, la enseñanza de la lengua debe estar orientada a establecer ciertas pautas de corrección lingüística impuestas por la gramática que se constituye en una ley que parte de los usos lingüísticos de las bellas letras y se aleja de la lengua de la comunidad. La lengua que se enseñe en la escuela debe borrar la pluralidad cultural para constituirse en algo fijo y puro.

Otro representante de esta tradición es Amado Alonso, profesor español que arriba a nuestro país en 1927 para asumir la dirección del Instituto de Filología de la Universidad de Buenos Aires, creado en 1923 por Ricardo Rojas. En su libro El problema argentino de la lengua (1935) deslegitima la existencia de un habla argentina y explicita que el problema argentino es la legitimación de lo no normal, de aquello que no responde a la norma. En una publicación posterior -La Argentina y la nivelación del idioma (1943)alerta sobre la situación lingüística porteña y plantea la necesidad de que en la escuela se enseñe el idioma patrio, especialmente en la escuela secundaria. Además, postula la necesidad de que cada nación tenga una lengua propia que es la que habla un pueblo y la de la literatura. Y agrega que si se considera lo propio como heredado, también la lengua española o castellana es propia de los argentinos porque la han heredado de generaciones anteriores. Por otro lado, plantea la tensión entre la lengua oral y la lengua literaria y considera que es la Academia quien debe fijar y ordenar lo que funciona como normal en la lengua literaria que es considerada el modelo a seguir. La lengua hablada, entonces, deberá tender hacia el ideal lingüístico presente en la literatura. Además, este autor explicita la necesidad de buscar "el progreso y la seguridad en el dominio del idioma" a través de la práctica de la ortología para corregir los "vulgarismos de pronunciación". En este sentido, en la Gramática castellana (1938) -escrita en colaboración con Pedro Henríquez Ureña- presenta "para su corrección muchas pronunciaciones defectuosas que son usuales" e invita a sus colegas a completar las listas con otros ejemplos habituales. Para Amado Alonso las formas correctas del lenguaje son aquéllas habladas por los grupos más cultos de la sociedad, es decir, la corrección lingüística implica el prestigio social de la cultura, las formas admitidas socialmente y distinguidas dentro del mercado 
lingüístico. Como modo de adiestrar a los alumnos en la lengua materna -que no es otra que el español castizo- propone las prácticas escolares de la conversación, la lectura literaria y la escritura. Para esto plantea el abordaje en la práctica oral de los "modos más propios, más ágiles y más expresivos del idioma, evitando a la vez la chabacanería y la afectación"; en la lectura el reconocimiento de "la significación y el valor estético de las palabras y de los giros del lenguaje artístico clásico y moderno" y, en la escritura plantea que distingan "entre lo conversacional y lo literario; que sepan con todo discernimiento cuáles son los buenos modos de su lengua y cuáles no, quedando avisados de los principales vulgarismos" y, por último, hace hincapié en la ortografía para que "les salga correcta como a un violinista le sale un nota afinada porque el dedo se le va al lugar justo de la cuerda" (7). La clase de Idioma patrio es el contexto en el cual se propone llevar a cabo estas prácticas con el fin de adquirir la lengua de la tradición literaria, aquélla que asegura un uso correcto del lenguaje en la vida cotidiana y la unidad lingüística hispanoamericana. Propone que sea el espacio escolar donde se aprenda la lengua literaria sin abandonar la lengua oral -propia de los intercambios lingüísticos familiares- que es la que da soltura al hablante. Para 1940, Alonso revisa sus concepciones en torno a la lengua $y$, si bien sigue estando presente una perspectiva aristocratizante y considera a la literatura como lugar de la norma, amplía su mirada reconociendo que la lengua general se constituye no sólo en la industria del libro sino también en otras esferas de lo social como la radio, las revistas, los periódicos, el cine.

El interés en establecer una lengua escolar libre de contaminaciones también está presente en la propuesta de Isaac B. Pearson, en el manual titulado Curso de castellano, publicado en la década del '30 para uso de alumnos de primer año de colegios nacionales, escuelas normales, liceos y escuelas de comercio e industriales, que fue utilizado incluso en la década del '50. En este manual -organizado en bolillas subdivididas en treinta lecciones y un "Apéndice para los estudiantes de las escuelas nacionales de comercio e industriales"- Pearson explicita la importancia de la gramática porque considera que "todo idioma pide fijeza" y, de esta manera, no habrá dificultades para la comunicación. Por otro lado, promueve el estudio de la etimología ya que establece cuáles son las normas lingüísticas que permiten "hablar con propiedad y escribir correctamente una lengua". Sin bien en la lección 1 se explicita que el propósito del libro es que los alumnos aprendan el idioma en el uso, lo lean "ejercitándose en la comprensión exacta del sentido de las palabras y oraciones", lo hablen "acostumbrándose a traducir con el lenguaje articulado sus ideas y sentimientos" y lo escriban "como 
se le debe escribir", el manual propone en cada lección la lectura de un trozo selecto, la realización de una síntesis, un cuestionario que apunta a la lectura literal y a la explicación del vocabulario. Otras de las consignas que se repiten en este manual es "escribir al dictado" y también se presentan cuadros explicativos "para la corrección de acentuaciones viciosas" o "para la corrección de los vicios más corrientes en la pronunciación de las consonantes" en diálogo con la creencia de que el elemento disruptivo en la lengua genera comportamientos lingüísticos que rompen con la moralidad del idioma y, por ello, son considerados vicios. Algunos ejemplos de usos incorrectos son: a) la supresión de una de las consonantes -uso lingüístico propio de los inmigrantes italianos- como, por ejemplo, "dotor" en lugar de "doctor", "adotar" en vez de "adoptar", "cospirar" en vez de "conspirar"; b) la supresión de una de las consonantes como, por ejemplo, "ojeto" en lugar de "objeto", "acaucil" en vez de "alcaucil"; c) la supresión de una de las consonantes y el agregado de otra como, por ejemplo, "ajetivo" en vez de "adjetivo", "rajuño" en lugar de "rasguño"; d) cambio de colocación de una consonantes como, por ejemplo, "itsmo" en lugar de "istmo"; e) supresión de la "s" final como, por ejemplo, "salimo junto lo tre" en lugar de "salimos juntos los tres", entre otros casos. De allí que el canon literario propuesto por Pearson -con el objetivo de ser recitado por el lector alumno- está conformado por un corpus de textos de autores como Ricardo Rojas, Leopoldo Díaz, Rubén Darío, Belisario Roldán, Juana de Ibarbouru, Arturo Capdevila que utilizan un lenguaje considerado correcto, sin vulgarismos y escriben sobre la riqueza y belleza de nuestro país.

Esta preocupación por corregir los usos "incorrectos" del lenguaje estuvo presente en las escuelas secundarias y primarias en los primeros años del siglo por ello se crearon asignaturas como Declamación, Oratoria, Recitado donde se estudiaba la prosodia u ortología con el objetivo de que los alumnos recitaran textos literarios o utilizaran correctamente la lengua oral. Para llevar adelante la práctica del recitado de textos literarios se conforma un canon literario ad hoc predominantemente con textos poéticos que representaban la norma estética literaria que ratificaba la norma lingüística como se puede observar en antologías y trozos selectos para uso escolar. Las antologías incluían textos de autores del panteón literario argentino, de la literatura hispánica o de la literatura universal considerados imprescindibles para la formación literaria del estudiante por su riqueza lingüística o patriótica; de esta manera, se construía una selección literaria que incluía a ciertos textos y excluía a otros y, además, el antólogo intervenía en cómo iban a ser leídos los textos. Una antología que se destaca en la diversidad que circulaban para las primeras décadas del siglo XX es Trozos 
escogidos de literatura y método de composición literaria (1904) de Alfredo Cosson, organizada en tres tomos en los cuales se incluyen textos literarios que son utilizados como modelos para la escritura de diversos géneros enmarcados en la retórica clásica como discursos, disertaciones morales y filosóficas, diálogos y cartas, entre otros. Otra antología es Prosa selecta (1905) de Enrique García Velloso quien fuera profesor del Colegio Nacional de Buenos Aires y de la Escuela Normal de Profesores; los textos que se incluyen son de autores de distintas nacionalidades y se proponen para el deleite y el aprendizaje del alumno. Otro texto es Lecturas selectas de Calixto Oyuela, publicado a principios del siglo, que incluye textos en prosa y en verso con intenciones historiográficas y universalistas. Avanzando el siglo, otro ejemplo de antología es Isondú (1927) de Correa Morales donde se seleccionan textos de autores argentinos que versan sobre temáticas nacionales y se proponen como modelos literarios para que los alumnos escriban sus propias composiciones. El autor, en el prólogo, explica que se propuso seleccionar textos que pueden ser dificultosos para los jóvenes por el vocabulario porque la lengua que utilizan los lectores es vulgar y "está llena de modismos e incorrecciones propios de nuestro país" (8). Se trata, entonces, de incluir textos de literatura pura para la formación lingüística y literaria de los alumnos. O, como señala el profesor José Fernández Coria -profesor en la Escuela Normal de maestros de Chivilcoy- en su libro La enseñanza de la literatura en las escuelas argentinas (1918) los textos deben promover la iniciación literaria y, para ello, es necesario seleccionar cuidadosamente los textos; por esto, sugiere textos de autores argentinos que el lector pueda sentir y "asimilar" como Olegario Andrade, Sarmiento, Obligado o Campoamor.

Una práctica escolar habitual que se emparenta con la literatura como bellas letras y responde al ideal de que los textos literarios son intemporales y objetos del deleite estético es la lectura artística o expresiva. Víctor Mercante en su libro Charlas pedagógicas (1925) promueve este tipo de lectura porque considera que sensibiliza al lector en los valores estéticos y morales de la literatura y, a su vez, permite que el alumno adquiera una doble dimensión: la de lector y la de artista. La lectura de literatura, entonces, exige la práctica de ejercicios de la voz, pronunciación y articulación y la imitación del alumno de la lectura modélica por parte del docente; asimismo, leer expresivamente implica un entrenamiento de la voz y físico como si se tratara de un actor ya que esta lectura atiende a los gestos, modales y posturas del que lee y, de esta manera, establece una hexis (9) corporal determinada que disciplina no sólo lingüísticamente sino también corporalmente al lector. En este sentido, Ricardo Monner Sans explicita en su 
Gramática castellana (1893) la "Reglas para leer bien." Para este profesor "la lectura en alta voz es la reproducción en sonidos claros y harmoniosos de las palabras y pensamientos expresados por medio de signos o letras"; para esto considera que "no basta (...) pronunciar con voz clara y armoniosa, dando a las palabras la entonación e inflexiones que requieran según expresan ideas de dolor o de alegría, de dulzura o de cólera" es necesario, "tener presente, al mismo tiempo, la posición que el cuerpo debe guardar, y la manera de moverse y accionar, que es lo que constituye el gesto." Además, agrega, "fuera de las pausas que indican los signos de puntuación mencionados, es necesario hacer otras para tomar aliento, evitando siempre trocar el sentido de la frase" y, por último, explica que los géneros literarios "requieren un tono y acento diferentes, y sólo mediante continuados ejercicios se llega a dar a la voz la graduación apropiada al asunto de la composición que se lee". En entrevistas a lectores que asistieron a la escuela en las primeras décadas del siglo, la lectura expresiva se recuerda como una práctica donde "el maestro hacía la lectura modelo y, luego, los alumnos teníamos que leer al frente, bien parados al lado del banco, con la mano izquierda sosteníamos el libro y con la derecha la hoja. Había que respetar esa postura y prestar atención a la pronunciación". La lectura expresiva o artística se pone al servicio del disciplinamiento lingüístico y de la domesticación corporal de los alumnos en consonancia con la tradición escolar que se propone erigir una lengua pura.

La búsqueda por instaurar el uso de una lengua escolar que erradique los usos populares se va constituyendo, a través de los años, en una tradición escolar que se nutre de distintas propuestas áulicas que dan cuenta de una concepción lingüística purista. No sólo se observa esta concepción de la enseñanza de la lengua en gramáticas y manuales sino también -avanzando el siglo XX- aparecen en este territorio textos que se proponen como guías para el uso del lenguaje, tanto en el ámbito escolar como más allá de su frontera. El profesor Gerardo Schiaffino publica en 1952 el texto Cómo hablamos... y cómo debemos hablar que se constituye en un compendio de dudas gramaticales y clasificación de vicios del lenguaje con su correcta utilización lingüística para uso del profesor y del alumno. Otro ejemplo es el texto Secretos y sorpresas del idioma (1961) de Luis Canossa considerado por su autor como "zarandeo lingüístico" que tiene como objetivo servir de "provecho y solaz a (...) todos cuantos sienten la noble inquietud de defender la pureza del sin par idioma de Cervantes". Si bien el texto no fue producido para el circuito escolar específicamente, es sugerido como lectura complementaria -como recuerda un entrevistado italiano, en ese entonces alumno de la escuela secundaria- por los profe- 
sores de Lengua por considerarlo una guía imprescindible para borrar de su lenguaje los "barbarismos fruto de su origen extranjero".

Para la misma época el Ministerio de Educación y Justicia impulsó la creación de un programa denominado Plan de Enseñanza y Moralidad del idioma que se proponía prevenir "el mal lenguaje difundido en la población escolar" y "contribuir a la limpieza de la palabra en toda su aplicación cultural y docente" siguiendo las normas de la lengua nacional. Avelino Herrero Mayor, profesor y filólogo español, forma parte de este programa y adhiere a la creencia de que es necesario reivindicar la moral del idioma y purificarlo. Publica en 1967 Diálogo argentino de la lengua. Cien lecciones para hablar bien y escribir mejor, estructurado como un diálogo socrático entre un profesor y una alumna en el que se plantean cuestiones referidas al uso del lenguaje y a temáticas gramaticales ejemplificadas con textos literarios del Siglo de Oro y vigiladas por la normativa de la Real Academia Española. El autor se propone "fomentar el conocimiento del idioma que nos fuera legado por la madre patria, y el conocimiento también de sus deformaciones, a fin de mantener la pureza de la lengua, incluso en lo que tiene de evolución propia y formación nacional" siguiendo el modelo del Diálogo de la lengua de Juan de Valdés y cuyo propósito era "adecentar gramatical y estéticamente nuestro lenguaje con consejos apropiados para hablarlo bien". Se trata, entonces, de reivindicar el "sentido castizo del habla criolla, coloreada de acento vernáculo" pero cuidando la gramática. En este sentido también advierte sobre los peligros de usar palabras extranjeras porque atentan "contra la unidad lingüística" y, por esto, sugiere no "emplear voces foráneas que no se conocen bien" porque da cuenta de un "desconocimiento del idioma propio". Este profesor no sólo alerta sobre los usos lingüísticos extranjeros, las consecuencias del cosmopolitismo en el lenguaje y la necesidad de respetar el idioma cervantino sino también le da suma importancia a la "pulcritud lingüística", es decir, "propiedad y decoro expresivos" para evitar "un descendimiento idiomático" y mostrar que "el buen lenguaje evidencia limpieza de alma". En otras palabras, para Herrero Mayor el lenguaje está ligado a principios morales que rigen la vida de los sujetos como hablantes y usuarios de la lengua y, por esto, considera imperativo promover la pureza lingüística como prescripción ministerial. Esta propuesta se completa con el folleto titulado El escritor y la palabra (1965), también publicado en el marco del Plan de enseñanza y moralidad del idioma, en el que Herrero Mayor presenta una serie de reflexiones sobre el uso del lenguaje para mejorar la expresión escrita.

En la historia de la tradición escolar en defensa de la limpieza y pureza del idioma otro hito va a ser el estructuralismo que se inicia en 1956 en la 
cátedra de Gramática de la Facultad de Filosofía y Letras de la UBA creada por Ana María Barrenechea (cátedra que luego de la represión ejercida en la llamada "Noche de los bastones largos" en 1966 queda a cargo de Ofelia Kovacci) y se arraiga como paradigma hegemónico, productor de prácticas escolares y rutinas didácticas cristalizadas en las escuelas secundarias de nuestro país que se instituyeron en las aulas y que perviven en el mapa escolar actual. En 1961 Mabel Manacorda de Rosetti publica La gramática estructural en la escuela secundaria, producto de una experiencia llevada a cabo en el Colegio Nacional de Buenos Aires, en el que presenta la teoría estructuralista tomando los aportes de Andrés Bello y Amado Alonso. Presenta este paradigma como un "método científico general" que estudia la sistematización de la lengua desde el criterio sintáctico. Por otro lado, explicita que la normativa gramatical determina la corrección o incorrección lingüística. En 1965 publica junto con María Hortensia Lacau, Castellano, donde vuelve sobre esta concepción de corrección lingüística a partir de la presentación de los tipos y niveles de lengua. Para estas autoras, el objetivo de la enseñanza es utilizar una lengua correcta -dada por el prestigio idiomático- para poder comunicarse. En este sentido, una práctica escolar habitual fue el análisis sintáctico de párrafos u oraciones de textos literarios "adecuados a los pasos graduales y que vayan presentando las grandes estructuras incluidas en ellas hasta llegar a (...) la palabra", separándolos en oraciones y luego entre sujeto y predicado y, dentro de éstos, los núcleos y modificadores. Otra rutina didáctica fue el análisis morfológico y semántico de palabras aisladas que se clasifican según el género y el número. En todos los casos se proponía que los ejercicios fueran "acompañados de observaciones teóricas, sintetizadas por escrito, paralelamente al desarrollo práctico y constantemente vinculadas con él". El análisis literario desde el paradigma estructuralista promovió el dispositivo escolar del cuestionario que apuntaba a reponer datos literales presentes en los textos y, como recuerda una profesora "el análisis de los textos literarios era a partir de preguntas sobre las figuras retóricas, el tema del texto, o bien preguntas acerca de si les gustaba el texto o no a los alumnos".

En la década del '70, esta tradición de la enseñanza de la lengua se mantiene y durante la última dictadura militar se promueven prácticas de raigambre purista -con el objetivo de normativizar el uso de la lengua- que se combinan con la tradición del estructuralismo. Como recuerda una profesora, "quien enseñara bien las subordinadas era un gran profesor", "no se daba la subordinada adverbial concesiva porque era la ideológica" y "la mejor manera de evadir el compromiso era dar mucha gramática, mucha normativa, bien rígida". 


\section{Conclusiones}

Si espiamos en aulas de escuelas primarias y secundarias podremos descubrir cómo los pizarrones acusan la presencia del análisis sintáctico estructuralista que convive con la clasificación de las variedades lingüísticas frente a la norma estándar y, a su vez, también está presente una enseñanza de la lengua ligada a perspectivas textualistas donde se plantea el imperativo de adecuar los textos a una norma lingüística o un formato estándar; es decir, aún hoy el discurso pedagógico se hace eco del mandato homogeneizador.

El idioma de Cervantes, entonces, funcionó en la historia de la disciplina escolar Lengua y Literatura como modelo y norma a seguir por su pureza y prestigio social; además, esa lengua libre de contaminaciones plurales se erigió como instrumento para morigerar las costumbres morales y sociales de la población escolar siguiendo una pretensión homogeneizadora.

La pregunta acerca de qué lengua debe enseñar la escuela, hoy sigue siendo motivo de disputas en el territorio escolar. Si bien múltiples voces confrontan en torno de cómo incorporar o excluir los lenguajes de la tecnología, qué posición tomar respecto de la interculturalidad producto de las migraciones y del lexicón de los jóvenes que pueblan las escuelas; el fantasma de la lengua cervantina como modelo lingüístico sigue estando presente en el discurso pedagógico -con matices más o menos homogeneizantes- y mantiene vigente esta tradición escolar.

\section{Notas Bibliográficas}

(1) Si bien Bourdieu toma el término "habitus" para explicar el comportamiento de un individuo como el producto de la aplicación de esquemas de percepción, estructuras estructurantes y apreciación de prácticas que producen diferencias y que son el resultado de las condiciones de existencia que se han atravesado, es decir, utiliza el término en un sentido abarcativo; aquí tomamos este concepto para explicitar cómo la imposición de la tradición escolar del purismo lingüístico trajo como consecuencia prácticas escolares que se proponían erradicar la relación que los inmigrantes tenían con su lengua y su cultura, como desalentar la pluralidad lingüística e imponer la monoglosia. Véase Bourdieu, P. (1997).

(2) Goodson, I. (1995).

(3) Cuesta Fernández, R. (1997), p. 21.

(4) Monner Sans, R. (1919), p. 54.

(5) Monner Sans, R. (1915), p. 186.

(6) Monner Sans, R. (1919), p. 359.

(7) Alonso, A. (1943), p. 102.

(8) Correa Morales (1927), p. X.

(9) Bourdieu, P. y Saint-Martin, M. (1975). 


\section{Referencias Bibliográficas}

- Alonso, A. (1935) "El problema argentino de la lengua", en El problema de la lengua en América. Madrid, Espasa - Calpe.

- Alonso, A. (1943) La Argentina y la nivelación del idioma. Buenos Aires, Institución Cultural Española.

- $\quad$ Alonso, A. y Henríquez Ureña, P. (1981) Gramática castellana. Segundo curso, Buenos Aires, Losada.

- $\quad$ Alonso, A. y Henríquez Ureña, P. (1983) Gramática castellana. Primer curso. Buenos Aires, Losada.

- Bombini, G. (2004) Los arrabales de la literatura. La historia de la enseñanza literaria en la escuela secundaria argentina (1880-1960). Buenos Aires, Miño \& Dávila.

- Bourdieu, P. (1997) Capital cultural, escuela y espacio social, Buenos Aires, Siglo Veintiuno editores.

- Bourdieu, P. y Saint Martin, M. "Las categorías del juicio profesoral", en Actes de la Recherche en Sciences Sociales, № 3, Paris, 1975. Traducción Emilio Tenti Fanfani.

- Canossa, L. (1961) Secretos y sorpresas del idioma. Buenos Aires, Editorial Atlántida.

- Cosson, A. (1904) Trozos escogidos de literatura y método de composición literaria, Buenos Aires, Librería Rivadavia.

- Cuesta Fernández, R. (1997) Sociogénesis de una disciplina escolar: la Historia. Barcelona, Ediciones Pomares-Corredor.

- Correa Morales, E. (1927) Isondú. Lecturas variadas para las escuelas comunes. Buenos Aires, Cabaut y Cía. Editores.

- Fernández Coria, J. (1918) La enseñanza de la literatura en las escuelas argentinas. Buenos Aires, Edición de la Revista "Nosotros."

- García Velloso, E. (1905) Prosa selecta. Buenos Aires, Ángel Estrada editores.

- Goodson, I. (1995) Historia crítica del currículum. Barcelona, Ediciones Pomares-Corredor.

- Herrero Mayor, A. (1965) El escritor y la palabra. Buenos Aires, Ministerio de Educación y Justicia.

- (1967) Diálogo argentino de la lengua. 100 lecciones para hablar bien y escribir mejor. Buenos Aires, Secretaría de Estado de Cultura y Educación.

- Lacau, M. H. y Manacorda de Rosetti, M. (1965) Castellano. Tercer año. Buenos Aires, Kapelusz.

- Manacorda de Rosetti, M. (1961) La gramática estructural en la escuela secundaria. Buenos Aires, Kapelusz.

- Mercante, V. (1925) Charlas pedagógicas 1890-1920. Buenos Aires, Gleizer editor.

- Monner Sans, R. (1913) Gramática castellana I. Prosodia y ortografía. Buenos Aires, Lajouane \& Cía. Editores. 
- Monner Sans, R. (1915) De gramática y de lenguaje. Madrid. Buenos Aires, Sucesores de Hernando - Cabaut.

- Monner Sans, R. (1919) Gramática castellana. Buenos Aires, Ángel Estrada editores.

- $\quad$ Oyuela, C. (s/r) Lecturas selectas. Buenos Aires, Ángel Estrada editores.

- Pearson, I. (s/r) Curso de castellano. Buenos Aires, Moly \& Laserre.

- Sardi, V. (2006) Historia de la lengua y la literatura. Continuidades y rupturas. Buenos Aires, Libros del Zorzal.

- Schiaffino, G. (1952) Cómo hablamos... y cómo debemos hablar. Buenos Aires, Editorial Bell. 